\title{
Competitive Hole Transfer from CdSe Quantum Dots to Thiol Ligands in CdSe- Cobaloxime Sensitized NiO Films Used as Photocathodes for $\mathrm{H}_{2}$ Evolution
}

\author{
Mohamed Abdellah, ${ }^{* \dagger, \ddagger \subset}$ Shihuai Zhang, ${ }^{\S}$ Mei Wang, ${ }^{\S}$ and Leif Hammarström*,† \\ ${ }^{\dagger}$ Department of Chemistry - Ångström Laboratory, Uppsala University, Box 523, 75120 Uppsala, Sweden \\ ${ }^{\ddagger}$ Department of Chemistry, Qena Faculty of Science, South Valley University, 83523 Qena, Egypt \\ ${ }^{\S}$ State Key Laboratory of Fine Chemicals, DUT-KTH Joint Education and Research Center on Molecular Devices, Dalian University \\ of Technology, Dalian 116024, China
}

\section{Supporting Information}

ABSTRACT: Quantum dot (QD) sensitized NiO photocathodes rely on efficient photoinduced hole injection into the $\mathrm{NiO}$ valence band. A system of a mesoporous $\mathrm{NiO}$ film co-sensitized with CdSe QDs and a molecular protonreduction catalyst was studied. While successful electron transfer from the excited QDs to the catalyst is observed, most of the photogenerated holes are instead quenched very rapidly (ps) by hole trapping at the surface thiols of the capping agent used as linker molecules. We confirmed our conclusion by first using a thiol free capping agent and second varying the thiol concentration on the QD's surface. The later resulted in faster hole trapping as the thiol concentration increased. We suggest that this hole trapping by the linker limits the $\mathrm{H}_{2}$ yield for this photocathode in a device.

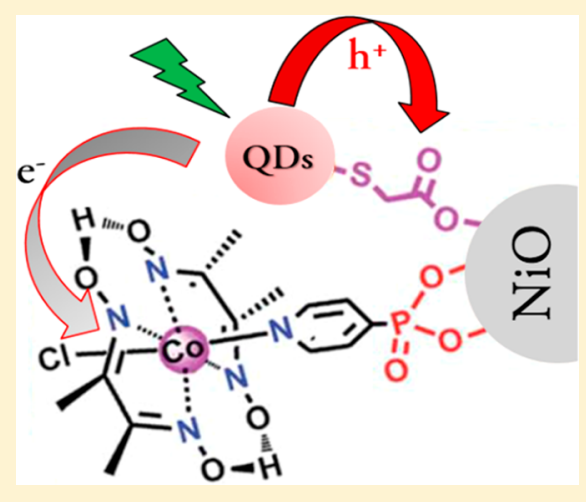

$\mathrm{V}$ isible-light-driven $\mathrm{H}_{2}$ evolution has been attracting considerable attention from both environmental and energy points of view and also as a scientific challenge in the artificial photosynthesis community. ${ }^{1-7}$ Many systems have been employed to produce $\mathrm{H}_{2}$ from sunlight-water splitting in a similar way to the natural photosynthetic systems. $^{4-7}$ In general, these systems contain a combination of light harvesting material and an active catalyst that is able to produce $\mathrm{H}_{2}$ after charge transfer from the light harvester. Therefore, a good understanding of the factors limiting photoinduced charge separation is needed to improve the catalytic activity of these systems.

Semiconductors quantum dots (QDs) like CdSe or CdS are popular sensitizers for solar energy applications including solar fuels. ${ }^{8,9}$ Here we report the photoinduced charge dynamics of both electrons and holes in an artificial system for $\mathrm{H}_{2}$ evolution that contains CdSe QDs as the light harvester and the wellknown molecular $\mathrm{CoP}$ catalyst $\left(\left[\mathrm{CoCl}(\mathrm{dmgH})_{2}\right.\right.$ (pyridyl-4hydrophosphonate)]) (Figure 1). Both the QDs and the CoP are chemically attached to a mesoporous $\mathrm{NiO}$ film via thioglycolic acid (TGA) and phosphonate groups, respectively. This system was reported recently by some of us as an efficient and stable photocathode for $\mathrm{H}_{2}$ production. ${ }^{4}$
CdSe QDs have been chosen as light harvesters due to their high extinction coefficient, broad absorption spectrum, sizedependent tunability of the band gap, and relatively simple preparation methods. Moreover, the $\mathrm{CoP}$ catalyst belongs to the best transition metal complexes known to produce $\mathrm{H}_{2}$ and is also easy to prepare. ${ }^{10}$ Herein, we investigate the photoinduced charge transfer dynamics upon excitation in this photocathode and find evidence for ultrafast hole transfer reactions that compete with productive hole injection into $\mathrm{NiO}$.

A one-pot adsorption and reaction (OPAR) method was used to prepare the TGA-capped CdSe QDs (see the SI for more details), while the CoP catalyst was prepared according to the published procedure. ${ }^{11}$ In this Letter, we find that the capping agent (TGA) of the QDs works as a trapping site for the photogenerated holes by using femtosecond transient absorption (TA) spectroscopy in the visible region. While the electrons are transferred from the excited QDs to the CoP catalyst as intended, most of the holes are trapped by the thiol groups and/or sulfide ions from the capping agent. We

Received: August 11, 2017

Accepted: October 16, 2017

Published: October 16, 2017 


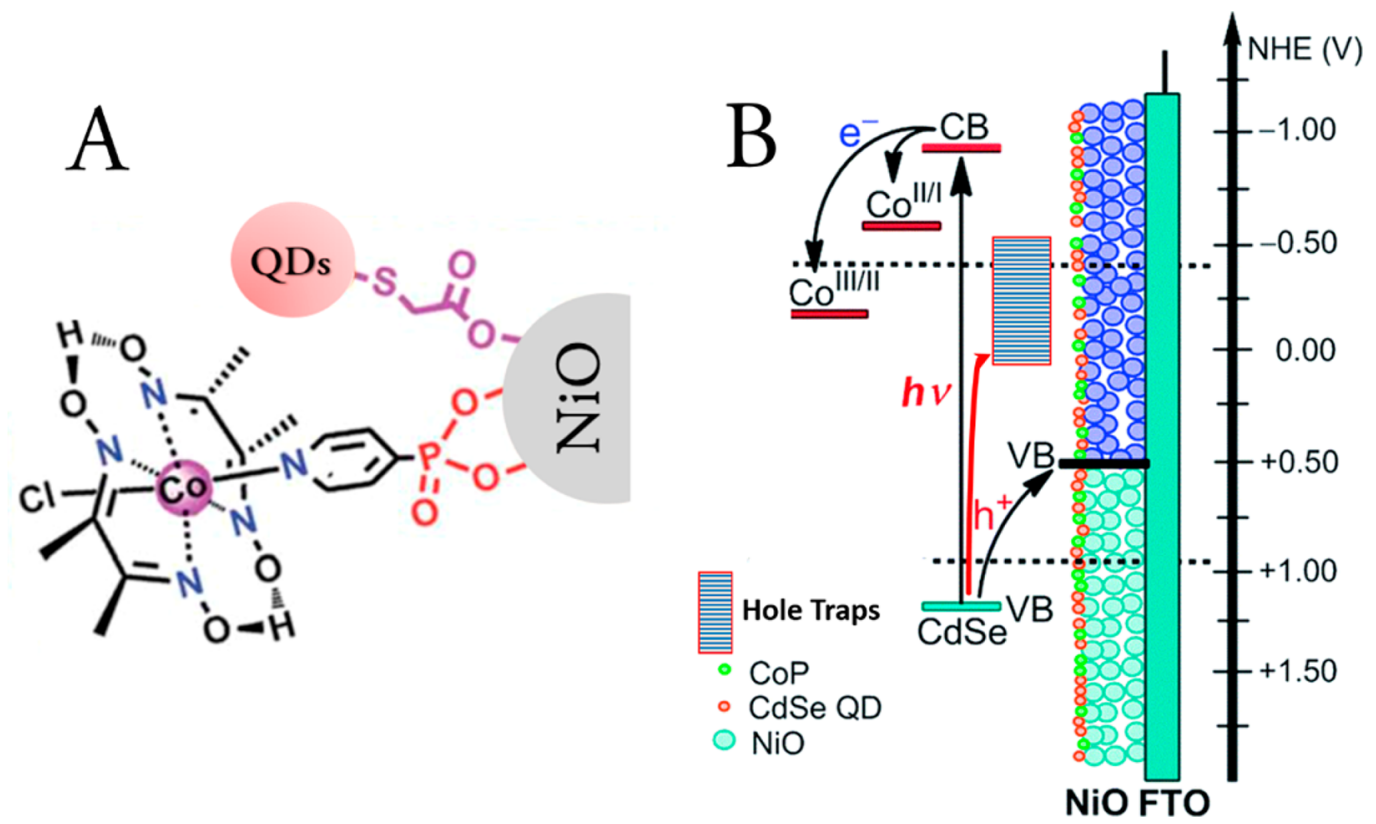

Figure 1. (A) Schematic presentation of the photocathode NiO/CdSe/CoP. QDs are attached to NiO through a TGA capping agent, while CoP molecules are attached through the phosphonate group. (B) Schematic presentation of the potentials of the system components; the surface hole trap states are assigned to the thiol linkers (see the main text).

compared the TGA-capped OPAR-CdSe QDs with thiol-free CdSe QDs capped with oleic acid that are prepared via hot injection (HI) method as a reference sample (CdSe HIQDs). ${ }^{12,13}$ Moreover, we compared TGA-capped OPAR-CdSe QDs with successive ionic layer adsorption and reaction (SILAR-CdSe QDs), both on $\mathrm{NiO}$, where the latter was reported to result in a lower catalytic activity in a device. ${ }^{4}$

Figure 2 presents steady-state absorption spectra and confirms the successful attachment of the OPAR-CdSe QDs

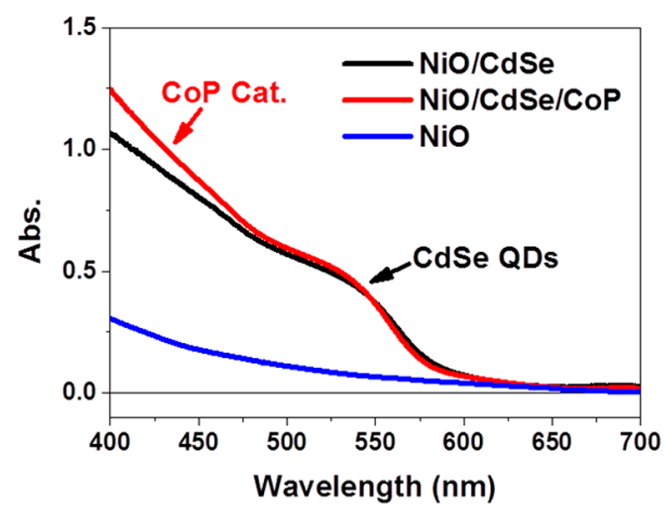

Figure 2. Steady-state UV-vis absorption spectra of the neat $\mathrm{NiO}$ film (blue line), $\mathrm{NiO} / \mathrm{CdSe}$ QDs film (black line), and the full photocathode $\mathrm{NiO} / \mathrm{CdSe} / \mathrm{CoP}$ (red line).

and the CoP catalyst onto the $\mathrm{NiO}$ surface, (red line; see the SI for more information about the sensitization process). The first (1S) peak ${ }^{13}$ of CdSe QDs (black line) is unchanged by introducing the catalyst (red line). An additional absorption feature at around $400 \mathrm{~nm}$ is due to the CoP attachement. ${ }^{14}$

Traps in CdS and CdSe QDs are intensively discussed in the literature ${ }^{15,16}$ due to their importance, ${ }^{17}$ but their origin is still not very clear. ${ }^{18}$ Energetically, it has been reported that the holes can be trapped in CdSe or CdTe QDs after excitiation. $^{18-20}$ This means that hole injection to a p-type semiconductor ( $\mathrm{NiO}$ in our system) and hole trapping processes are difficult to distinguish spectroscopically where both have the same signature of charge depopulation. ${ }^{13,21}$ Herein, we will discuss the electron transfer from excited QDs to the catalyst and the self-hole trapping process by a TGA capping agent for TGA-capped CdSe QDs in a $\mathrm{NiO} / \mathrm{CdSe} /$ CoP photocathode.

Figure 3A,C presents the TA spectra of the $\mathrm{NiO} / \mathrm{CdSe}$ film and $\mathrm{NiO} / \mathrm{CdSe} / \mathrm{CoP}$ film, respectively, at indicated time delays after the $400 \mathrm{~nm}$ excitation pulse. Generally, the spectra of excited and reduced QDs look similar, ${ }^{9}$ while oxidation of QDs leads to faster recovery of the ground-state bleach (GSB) as GSB is mainly due to electron population in the conduction band. ${ }^{12}$ In Figure 3A, a bleach at $\sim 570 \mathrm{~nm}$ is seen due to the first exciton absorption band of CdSe QDs (state filling of the $1 S$ exciton). ${ }^{12,22}$ On the lower-energy side of the GSB (600$700 \mathrm{~nm}$ ), there is strong positive absorption due to the trapped carriers, as has been reported before for CdTe QDs and CdTe/ CdS core/shell QDs. ${ }^{23,24}$ To exclude any other carrier interactions that may cause this positive absorption in our system, like biexcitonic effects, we used a low pump intensity, which led to $N<1$ ( $N$ is the average number of electron-hole pairs per QD). ${ }^{12,22}$

Even though the TA spectra of $\mathrm{NiO} / \mathrm{CdSe} \mathrm{QD}$ systems with and without CoP look similar, the GSB recovery dynamics of both systems are different (Figure 3B,D, black lines). Introducing $\mathrm{CoP}$ catalyst accelerates the GSB recovery, meaning that the CoP catalyst consumes electrons from the excited QDs to form the reduced Co ${ }^{\mathrm{II}}$. It was not possible to spectroscopically detect the comparably weak feature of the reduced CoP catalyst species $\left(\mathrm{Co}^{\mathrm{II}}\right)$ at around $450 \mathrm{~nm}$ as it was observed before ${ }^{25}$ due to the positive absorption features from other species in the entire visible region, as will be discussed later, and due to its lower extinction coefficient of these species. $^{26}$

Comparing the GSB recovery kinetics confirms the electron transfer from excited QDs to CoP catalyst. ${ }^{14}$ Figure 3B,D 

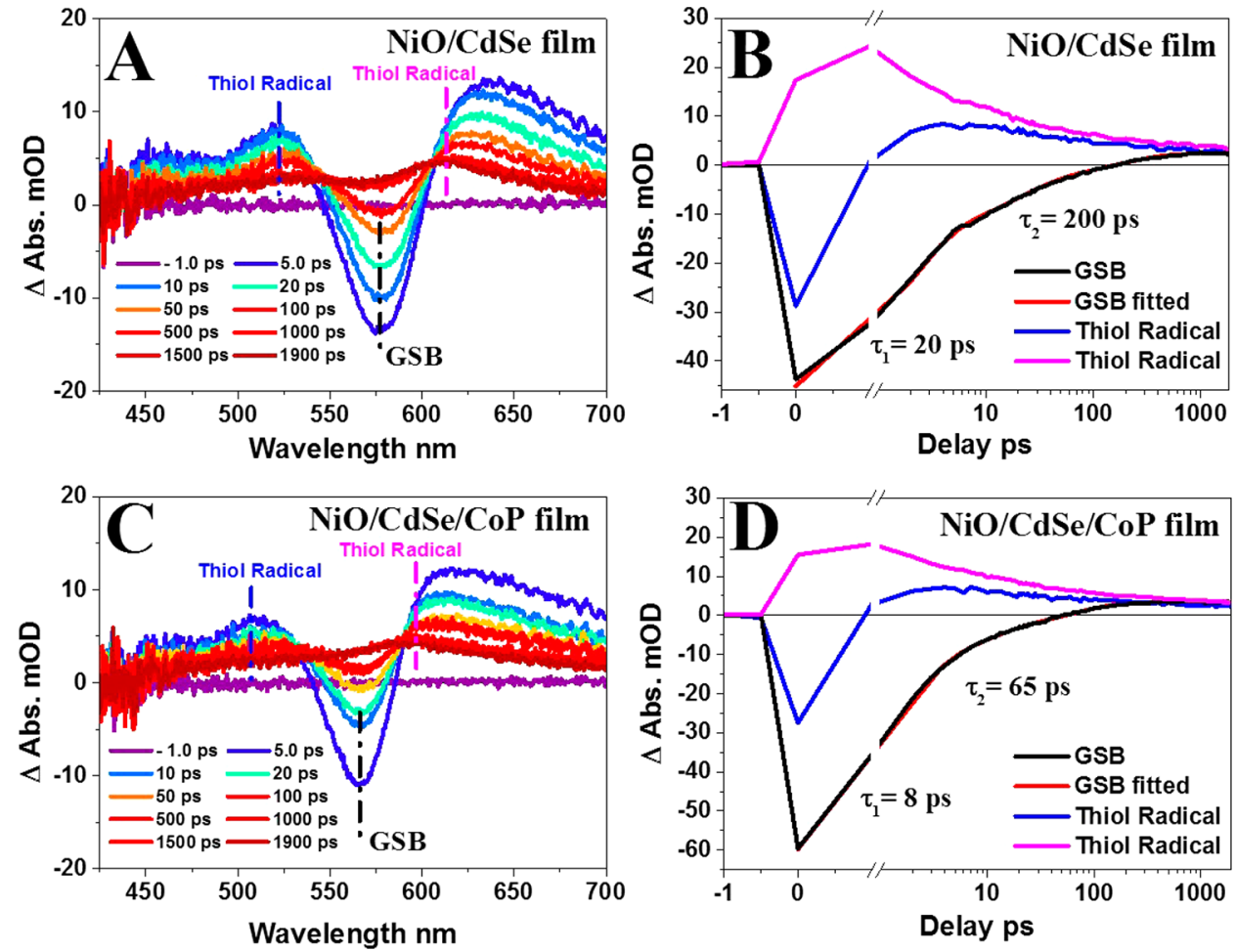

Figure 3. TA spectra for the $\mathrm{NiO} / \mathrm{CdSe}$ film without $(\mathrm{A})$ and with $(\mathrm{C})$ the introduction of $\mathrm{CoP}$ catalyst. TA traces for $\mathrm{NiO} / \mathrm{CdSe}$ film without (B) and with (D) the introduction of CoP catalyst; black lines present the GSB recovery, and red lines present the fitting of the GSB recovery traces.

shows the comparison of the GSB recovery (black lines) for $\mathrm{NiO} / \mathrm{CdSe}$ (panel B) $\left(\tau_{1}=20 \mathrm{ps}, A_{1}=65 \%\right.$ and $\tau_{2}=200 \mathrm{ps}, A_{2}$ $=34 \%)$ and $\mathrm{NiO} / \mathrm{CdSe} / \mathrm{CoP}$ (panel D) $\left(\tau_{1}=8 \mathrm{ps}, A_{1}=77 \%\right.$ and $\left.\tau_{2}=65 \mathrm{ps}, A_{2}=23 \%\right)$. The biexponential fit reflects the typical heterogeneity of interfacial electron transfer and should not be interpreted as two distinct processes. It is well-known that the exciton lifetime of CdSe QDs (HI-QDs) is around 10$20 \mathrm{~ns},{ }^{20}$ while for the OPAR QDs, the exciton lifetime is very short (see Figure S2E). The shorter exciton lifetime is due to the presence of trap states, which generally affects the electron dynamics.

One important question to be answered is what is the destiny of the photogenerated holes? $\mathrm{H}_{2}$ production quantum yields under continuous irradiation are dependent on the reactions of the remaining holes in the QDs after the transfer of the electron to the catalyst. ${ }^{21}$ In the ideal case scenario, as both CdSe QDs and $\mathrm{CoP}$ are chemically attached to $\mathrm{NiO}$ surface, the holes should end up in the valence band of $\mathrm{NiO}^{27}$ and then migrate to the external circuit. However, we found that most of the photogenerated holes are trapped by the thiol groups and/or sulfide ions on a subps to ps time scale. This is seen from the rapid appearance of multiple absorption bands in the TA spectra (Figure 3A,C), where polythiol and polysulfide radicals are known to absorb. ${ }^{28-32}$ The absorption bands remain at $t>$ 1000 ps when all GSB is gone, which excludes assignment to a Stark effect. ${ }^{9,14}$ In the context of quantum dots sensitized solar cells (QDSSCs), it has been reported that hole scavenging by external scavengers, ${ }^{33}$ like the sulfide redox couple $\left(\mathrm{S}^{2-} / \mathrm{S}_{\mathrm{n}}{ }^{2-}\right)^{28}$ or sulfide salt as the ionic liquid, ${ }^{23}$ takes place on much slower time scales (tens of ns). In our system, we observe a much faster hole trapping process (ps) with a spectroscopic signature of di- and polysulfide radicals. ${ }^{28-30}$ Our TA spectra are in excellent agreement with those reported for CdSe QDs on
$\mathrm{TiO}_{2}$ in the presence of $\mathrm{NaS}_{2}$ on a ns $-\mu$ s time scale. ${ }^{28}$ On one hand, capping agents are used to protect the $\mathrm{QD}$ surface and to prevent QD aggregation. On the other hand, TGA is the only thiol source in our system, and formation of sulfide radicals suggests that thiol groups are preferentially oxidized instead of the $\mathrm{NiO}$ valence band (Figure $1 \mathrm{~B}$ ). The rapid oxidation may be due to the direct binding of the thiol groups to the QD, while external scavengers would be sterically hindered to approach the surface by capping agents. ${ }^{28,33}$ In our case, the ultrafast radical formation may compete with desired hole transfer to NiO. To confirm our conclusion that the TA signals are related to TGA oxidation, we attached the OPAR-CdSe QDs to the surface of $\mathrm{ZrO}_{2}$ (noninjecting semiconductor) ${ }^{34,35}$ in the same manner of the $\mathrm{NiO}$ system without the catalyst. Both samples $\mathrm{NiO} / \mathrm{CdSe}$ (Figure 3A) and $\mathrm{ZrO}_{2} / \mathrm{CdSe}$ (Figure S2A) show similar TA spectra with strong positive absorption over the entire visible region due to the formation of di/polysulfide radicals. Moreover, we compare the TA traces of OPAR-QDs attached to $\mathrm{ZrO}_{2}$ at the radical absorption peaks with the TA traces when the OPAR-QDs are attached to NiO (Figure S2BD); the TA traces look very similar when $\mathrm{ZrO}_{2}$ is replaced by $\mathrm{NiO}$. This shows that a major fraction of the photogenerated holes are trapped by the thiol groups of the linker instead of being directly injected into $\mathrm{NiO}$. It is difficult to quantify the ratio of hole transfer to TGA vs $\mathrm{NiO}$ by comparing with reference samples as the TGA concentration varies between samples (TGA is added in a one-pot preparation). The radical $\mathrm{TA}$ signal rise is even somewhat slower on $\mathrm{NiO}$ than that on $\mathrm{ZrO}_{2}$ (Figure S2), which obviously cannot be explained by additional hole injection into $\mathrm{NiO}$. Comparison with hole injection into $\mathrm{NiO}$ for QDs prepared by other methods is also questionable as the electron transfer rate can be expected to vary between different QDs and with different linker groups. 
Furthermore, we measured the TA spectra of CdSe QDs prepared by the HI method (HI-QDs) and capped with a thiolfree capping agent, namely, oleic acid. The TA spectra did not show the spectroscopic signature of polythiol radicals and/or polysulfide radicals (see Figures 3A and S1A). To confirm that the formation of sulfuric radicals is due to the self-trapping of holes by the thiol groups on the QDs surface, we changed the capping agent of HI-QDs from oleic acid to TGA and attached the TGA-capped HI-QDs to a $\mathrm{NiO}$ film. The resulting TA spectra of this film indeed show similar positive absorption features on similar time scales (see Figure S1B,C) as those for the OPAR-CdSe samples in Figure 3. This confirms the involvement of the TGA capping agent in the hole trapping process. Figure 3B,D presents the traces of polythiols radical and/or polysulfide radical formation due to the oxidation of the thiol groups and/or sulfide ions by the photogenerated holes in the QDs. We also measured the TA of SILAR QDs that showed instantaneous trapping of the charge carriers upon excitation (Figure S3). This explains the poor catalytic activity of the SILAR QD devices, ${ }^{4}$ in addition to other problems like the poor loading on $\mathrm{NiO}$ compared to OPAR QDs.

Formation of the sulfuric radicals allows us to follow the dynamics of the photogenerated holes, but on the other hand, this trapping process competed with hole injection into $\mathrm{NiO}$ and is therefore likely to reduce the catalytic activity of a device. Thiol and sulfur reduction occur at mild potentials ${ }^{29,32,36}$ (cf. "Hole traps" in Figure 1B), and the resulting radicals can most likely not oxidize the $\mathrm{NiO}$ valence band ( $E \approx 0.5 \mathrm{~V}$ vs $\mathrm{NHE}){ }^{37}$ as has been reported in the best-case scenario. ${ }^{27}$ Although this option cannot be totally excluded in our system, we note that the radical decay kinetics on $\mathrm{NiO}$ and $\mathrm{ZrO}_{2}$ is essentially identical (cf. Figures 3 and S2). This suggests that at least most of the holes trapped on the radicals do not lead to $\mathrm{NiO}$ oxidation. Instead, the rapid quenching of the QDs by TGA reduces the photocurrent and efficiency of $\mathrm{H}_{2}$ evolution that can be achieved in a device.

Radical decay on $\mathrm{NiO}$ and $\mathrm{ZrO}_{2}$ is tentatively assigned to charge recombination with the reduced catalyst and/or radical reactions such as radical-radical coupling. We further note that irreversible radical reactions could make TGA a sacrificial donor. This could in principle lead to high quantum yields of hydrogen generation $\left(\Phi_{\mathrm{H} 2}\right)$ at the photocathode without the need for corresponding oxidation at the counter electrode, i.e., photochemical $\mathrm{H}_{2}$ without a corresponding photocurrent. This was not the case in ref 4 , however, as $\Phi_{\mathrm{H} 2}$ was small and the Faradaic efficiency was close to $100 \%$ (and not $\gg 100 \%$ ). ${ }^{4}$

The present study has shown that the capping agent (TGA), which protects the QDs from aggregation, itself can act as a hole trap and thus be a limiting factor for solar fuel application of QDs as a photosensitizer. Therefore, further treatments for the QDs can lead to better $\mathrm{H}_{2}$ evolution efficiency like using thiol-free capping agents or using a core/shell structure with an optimized shell thickness ${ }^{38}$ to reduce the hole trapping probability.

\section{ASSOCIATED CONTENT}

\section{S Supporting Information}

The Supporting Information is available free of charge on the ACS Publications website at DOI: 10.1021/acsenergylett.7b00730.

Synthesis of CdSe QDs and $\mathrm{NiO}$ and complementary transient absorption data (PDF)

\section{AUTHOR INFORMATION}

\section{Corresponding Authors}

*E-mail: Leif.Hammarstrom@kemi.uu.se (L.H.).

*E-mail: Mohamed.Qenawy@kemi.uu.se (M.A.).

ORCID

Mohamed Abdellah: 0000-0002-6875-5886

Leif Hammarström: 0000-0002-9933-9084

Notes

The authors declare no competing financial interest.

\section{ACKNOWLEDGMENTS}

We would like to acknowledge the Knut and Alice Wallenberg Foundation (2011.0067), the Swedish Energy Agency (116745), the Swedish Research Council (2014-5921), and the National Natural Science Foundation of China (Nos. 21373040 and 21673028) for financial support. Dr. Burkhard Zietz, Dr. Mélina Gilbert Gatty, Dr. Ahmed El-Zohry, and Jens Föhlinger are acknowledged for their help with the fs-TA setup. We are grateful to Minglun Cheng for preparation of the SILAR QD samples.

\section{REFERENCES}

(1) Lubitz, W.; Tumas, W. Hydrogen: An Overview. Chem. Rev. 2007, 107, 3900-3903.

(2) Youngblood, W. J.; Lee, S.-H. A.; Maeda, K.; Mallouk, T. E. Visible Light Water Splitting Using Dye-Sensitized Oxide Semiconductors. Acc. Chem. Res. 2009, 42, 1966-1973.

(3) Magnuson, A.; Anderlund, M.; Johansson, O.; Lindblad, P.; Lomoth, R.; Polivka, T.; Ott, S.; Stensiö, K.; Styring, S.; Sundström, V.; Hammarström, L. Biomimetic and Microbial Approaches to Solar Fuel Generation. Acc. Chem. Res. 2009, 42, 1899-1909.

(4) Meng, P.; Wang, M.; Yang, Y.; Zhang, S.; Sun, L. CdSe quantum dots/molecular cobalt catalyst co-grafted open porous $\mathrm{NiO}$ film as a photocathode for visible light driven $\mathrm{H}_{2}$ evolution from neutral water. J. Mater. Chem. A 2015, 3, 18852-18859.

(5) McKone, J. R.; Lewis, N. S.; Gray, H. B. Will Solar-Driven WaterSplitting Devices See the Light of Day? Chem. Mater. 2014, 26, 407414.

(6) Esswein, A. J.; Nocera, D. G. Hydrogen Production by Molecular Photocatalysis. Chem. Rev. 2007, 107, 4022-4047.

(7) Eckenhoff, W. T.; Eisenberg, R. Molecular systems for light driven hydrogen production. Dalton Trans. 2012, 41, 13004-13021.

(8) Bang, J. H.; Kamat, P. V. Quantum Dot Sensitized Solar Cells. A Tale of Two Semiconductor Nanocrystals: CdSe and CdTe. ACS Nano 2009, 3, 1467-1476.

(9) Wu, K.; Zhu, H.; Lian, T. Ultrafast Exciton Dynamics and LightDriven $\mathrm{H}_{2}$ Evolution in Colloidal Semiconductor Nanorods and PtTipped Nanorods. Acc. Chem. Res. 2015, 48, 851-859.

(10) Kaeffer, N.; Chavarot-Kerlidou, M.; Artero, V. Hydrogen Evolution Catalyzed by Cobalt Diimine-Dioxime Complexes. Acc. Chem. Res. 2015, 48, 1286-1295.

(11) Lakadamyali, F.; Reisner, E. Photocatalytic $\mathrm{H}_{2}$ evolution from neutral water with a molecular cobalt catalyst on a dye-sensitised $\mathrm{TiO}_{2}$ nanoparticle. Chem. Commun. 2011, 47, 1695-1697.

(12) Žídek, K.; Zheng, K.; Ponseca, C. S.; Messing, M. E.; Wallenberg, L. R.; Chábera, P.; Abdellah, M.; Sundström, V.; Pullerits, T. Electron Transfer in Quantum-Dot-Sensitized $\mathrm{ZnO}$ Nanowires: Ultrafast Time-Resolved Absorption and Terahertz Study. J. Am. Chem. Soc. 2012, 134, 12110-12117.

(13) Zheng, K.; Žídek, K.; Abdellah, M.; Zhang, W.; Chábera, P.; Lenngren, N.; Yartsev, A.; Pullerits, T. Ultrafast Charge Transfer from CdSe Quantum Dots to p-Type NiO: Hole Injection vs Hole Trapping. J. Phys. Chem. C 2014, 118, 18462-18471.

(14) Huang, J.; Mulfort, K. L.; Du, P.; Chen, L. X. Photodriven Charge Separation Dynamics in CdSe/ZnS Core/Shell Quantum 
Dot/Cobaloxime Hybrid for Efficient Hydrogen Production. J. Am. Chem. Soc. 2012, 134, 16472-16475.

(15) Jones, M.; Lo, S. S.; Scholes, G. D. Quantitative modeling of the role of surface traps in $\mathrm{CdSe} / \mathrm{CdS} / \mathrm{ZnS}$ nanocrystal photoluminescence decay dynamics. Proc. Natl. Acad. Sci. U. S. A. 2009, 106, 30113016.

(16) Wu, K.; Zhu, H.; Liu, Z.; Rodríguez-Córdoba, W.; Lian, T. Ultrafast Charge Separation and Long-Lived Charge Separated State in Photocatalytic CdS-Pt Nanorod Heterostructures. J. Am. Chem. Soc. 2012, 134, 10337-10340.

(17) Baker, D. R.; Kamat, P. V. Tuning the Emission of CdSe Quantum Dots by Controlled Trap Enhancement. Langmuir 2010, 26, 11272-11276.

(18) Utterback, J. K.; Grennell, A. N.; Wilker, M. B.; Pearce, O. M.; Eaves, J. D.; Dukovic, G. Observation of trapped-hole diffusion on the surfaces of CdS nanorods. Nat. Chem. 2016, 8, 1061-1066.

(19) Klimov, V. I. Spectral and dynamical properties of multiexcitons in semiconductor nanocrystals. Annu. Rev. Phys. Chem. 2007, 58, 635673.

(20) Abdellah, M.; Marschan, R.; Žídek, K.; Messing, M. E.; Abdelwahab, A.; Chábera, P.; Zheng, K.; Pullerits, T. Hole Trapping: The Critical Factor for Quantum Dot Sensitized Solar Cell Performance. J. Phys. Chem. C 2014, 118, 25802-25808.

(21) Yang, Y.; Wu, K.; Shabaev, A.; Efros, A. L.; Lian, T.; Beard, M. C. Direct Observation of Photoexcited Hole Localization in CdSe Nanorods. ACS En. Lett. 2016, 1, 76-81.

(22) Ž́ídek, K.; Zheng, K.; Abdellah, M.; Lenngren, N.; Chábera, P.; Pullerits, T. Ultrafast Dynamics of Multiple Exciton Harvesting in the $\mathrm{CdSe}-\mathrm{ZnO}$ System: Electron Injection versus Auger Recombination. Nano Lett. 2012, 12, 6393-6399.

(23) Sekhar, M. C.; Santhosh, K.; Praveen Kumar, J.; Mondal, N.; Soumya, S.; Samanta, A. CdTe Quantum Dots in Ionic Liquid: Stability and Hole Scavenging in the Presence of a Sulfide Salt. J. Phys. Chem. C 2014, 118, 18481-18487.

(24) Rawalekar, S.; Kaniyankandy, S.; Verma, S.; Ghosh, H. N. Ultrafast Charge Carrier Relaxation and Charge Transfer Dynamics of $\mathrm{CdTe} / \mathrm{CdS}$ Core-Shell Quantum Dots as Studied by Femtosecond Transient Absorption Spectroscopy. J. Phys. Chem. C 2010, 114, $1460-1466$.

(25) Du, P.; Schneider, J.; Luo, G.; Brennessel, W. W.; Eisenberg, R. Visible Light-Driven Hydrogen Production from Aqueous Protons Catalyzed by Molecular Cobaloxime Catalysts. Inorg. Chem. 2009, 48, $4952-4962$.

(26) Bacchi, M.; Berggren, G.; Niklas, J.; Veinberg, E.; Mara, M. W.; Shelby, M. L.; Poluektov, O. G.; Chen, L. X.; Tiede, D. M.; Cavazza, C.; Field, M. J.; Fontecave, M.; Artero, V. Cobaloxime-Based Artificial Hydrogenases. Inorg. Chem. 2014, 53, 8071-8082.

(27) Liu, B.; Li, X.-B.; Gao, Y.-J.; Li, Z.-J.; Meng, Q.-Y.; Tung, C.-H.; $\mathrm{Wu}, \mathrm{L} . \mathrm{Z}$. A solution-processed, mercaptoacetic acid-engineered CdSe quantum dot photocathode for efficient hydrogen production under visible light irradiation. Energy Environ. Sci. 2015, 8, 1443-1449.

(28) Chakrapani, V.; Baker, D.; Kamat, P. V. Understanding the Role of the Sulfide Redox Couple $\left(\mathrm{S}^{2-} / \mathrm{S}_{\mathrm{n}}{ }^{2-}\right)$ in Quantum Dot-Sensitized Solar Cells. J. Am. Chem. Soc. 2011, 133, 9607-9615.

(29) Asmus, K.-D. Sulfur-centered free radicals. Methods Enzymol. 1990, 186, 168-180.

(30) Asmus, K. D. Stabilization of oxidized sulfur centers in organic sulfides. Radical cations and odd-electron sulfur-sulfur bonds. Acc. Chem. Res. 1979, 12, 436-442.

(31) Chivers, T.; Drummond, I. Characterization of the trisulfur radical anion $\mathrm{S}^{3-}$ in blue solutions of alkali polysulfides in hexamethylphosphoramide. Inorg. Chem. 1972, 11, 2525-2527.

(32) Clark, R. J. H.; Cobbold, D. G. Characterization of sulfur radical anions in solutions of alkali polysulfides in dimethylformamide and hexamethylphosphoramide and in the solid state in ultramarine blue, green, and red. Inorg. Chem. 1978, 17, 3169-3174.

(33) Huang, J.; Huang, Z.; Jin, S.; Lian, T. Exciton Dissociation in CdSe Quantum Dots by Hole Transfer to Phenothiazine. J. Phys. Chem. C 2008, 112, 19734-19738.
(34) Corani, A.; Li, M.-H.; Shen, P.-S.; Chen, P.; Guo, T.-F.; El Nahhas, A.; Zheng, K.; Yartsev, A.; Sundström, V.; Ponseca, C. S. Ultrafast Dynamics of Hole Injection and Recombination in Organometal Halide Perovskite Using Nickel Oxide as p-Type Contact Electrode. J. Phys. Chem. Lett. 2016, 7, 1096-1101.

(35) Abdellah, M.; El-Zohry, A. M.; Antila, L. J.; Windle, C. D.; Reisner, E.; Hammarström, L. Time-Resolved IR Spectroscopy Reveals a Mechanism with $\mathrm{TiO}_{2}$ as a Reversible Electron Acceptor in a $\mathrm{TiO}_{2}-$ Re Catalyst System for $\mathrm{CO}_{2}$ Photoreduction. J. Am. Chem. Soc. 2017, 139, 1226-1232.

(36) Freedman, L. D.; Corwin, A. H. Oxidation-Reduction Potentials of Thiol-Disulfide Systems. J. Biol. Chem. 1949, 181, 601-621.

(37) Odobel, F.; Pellegrin, Y.; Gibson, E. A.; Hagfeldt, A.; Smeigh, A. L.; Hammarström, L. Recent advances and future directions to optimize the performances of p-type dye-sensitized solar cells. Coord. Chem. Rev. 2012, 256, 2414-2423.

(38) Abdellah, M.; Žídek, K.; Zheng, K.; Chábera, P.; Messing, M. E.; Pullerits, T. Balancing Electron Transfer and Surface Passivation in Gradient $\mathrm{CdSe} / \mathrm{ZnS}$ Core-Shell Quantum Dots Attached to ZnO. J. Phys. Chem. Lett. 2013, 4, 1760-1765. 